\title{
The effect of normal saline instillation on cardiorespiratory parameters in intubated cardiothoracic patients
}

\author{
S Schmollgruber, ' CCRN, MSc (Nursing), PhD(c); J C Bruce,' RN, MSc (Nursing), PhD; J C Rachidi,' MSc (Nursing); \\ P J Becker, ${ }^{1,2} \mathrm{MSc}, \mathrm{PhD}$ \\ ' Department of Nursing Education, Faculty of Health Sciences, University of the Witwatersrand, Johannesburg, South Africa \\ ${ }^{2}$ Biostatistics Unit, Medical Research Council, Pretoria, South Africa
}

Corresponding author: S Schmollgruber (shelley.schmollgruber@wits.ac.za)

\begin{abstract}
Objective. The objective of this study was to describe the effect of normal saline instillation (NSI) on cardiorespiratory parameters in intubated cardiothoracic patients.

Methods. A comparative design was employed to meet the study objectives. Simple random sampling was used to assign patients to study groups, namely a research group (non-NSI) and a control group (NSI). The data-capturing tool was based on the literature review. Descriptive and comparative statistics were employed to analyse the data. Findings were assessed according to $p<0.05$.

Results. Findings indicated that there were no statistically significant differences in heart rate, blood pressure, arterial partial pressure of oxygen $\left(\mathrm{PaO}_{2}\right)$, arterial oxygen saturation as measured by blood gas analyser $\left(\mathrm{SaO}_{2}\right)$ and serum bicarbonate level $\left(\mathrm{HCO}_{3}^{-}\right)$when $\mathrm{NSI}$ was used or not used during endotracheal suctioning $(p=0.05)$. Statistically significant differences were found in $\mathrm{pH}$ and patient return rate to baseline arterial oxygen saturation as measured by pulse oximetry $\left(\mathrm{SpO}_{2}\right)$ after 30 minutes of suctioning: $63.6 \%$ of patients in the NSI group failed to return to baseline $\mathrm{SpO}_{2}$ v. $37.5 \%$ of patients in the non-NSI group $\left(\chi^{2} p=0.035\right.$; Fisher's exact $\left.p=0.048\right)$. There was a decrease in $p H$ when NSI was used during suctioning. Although these differences were statistically significant, clinically they were not significant.

Conclusion. It can be concluded that NSI had no effect on cardiorespiratory parameters in intubated cardiothoracic patients. Even though the patient population was at high risk of haemodynamic disturbance and hypoxia during this manoeuvre, there was no meaningful clinical effect; however, the sample size was too small to establish safety.
\end{abstract}

S Afr J Crit Care 2014;30(1):22-27. DOI:10.7196/SAJCC.181

Endotracheal suctioning (ETS) is one of the most common procedures, routinely used in critical care to remove secretions that could potentially occlude the airway of critically ill and mechanically ventilated patients. The concomitant instillation of normal saline to enhance the effectiveness of suctioning has been debated for several years, ${ }^{[1]}$ highlighting the detrimental effects of normal saline instillation (NSI). NSI involves instilling a bolus of normal saline ranging from $0.25 \mathrm{~mL}$ to $10 \mathrm{~mL}$ into the endotracheal tube (ET) just before suctioning to facilitate removal of secretions, thus preventing tube occlusion and atelectasis or alveolar collapse and improving oxygenation. ${ }^{[2,3]}$ Furthermore, NSI reportedly eases catheter insertion, induces coughing and increases aspiration of secretions. ${ }^{[3]}$

However, the evidence suggests that NSI has adverse effects on oxygenation, level of dyspnoea, blood pressure (BP), heart rate (HR) and other vital parameters. ${ }^{[4,5]}$ The risks with NSI seem to outweigh the perceived benefit of increased removal of secretions. In contrast, there are some studies that point to the reduction/ no increase in ventilator-acquired pneumonia due to the effects of saline on the quality and quantity of secretions. ${ }^{[6,7]}$ There was, however, no difference in mortality, morbidity (atelectasis and endotracheal blockage), ventilator days and ICU length of stay. Increased frequency of nosocomial infections appears to be a better predictor of increased mortality, prolonged hospitalisation and increased healthcare costs. ${ }^{[8,9]}$ Subjective patient reports of increased pain, discomfort and dyspnoea, and feelings of anxiety, dread and drowning are well documented in the literature. ${ }^{[1,8,10]}$.
The theory behind the benefits of NSI is questionable and studies that have shown benefits of NSI for nursing practice have been criticised for methodological limitations and relevance of context. ${ }^{[4,11]}$ The balance of probability leans towards more harm than good, but despite this NSI continues to be used in ICUs locally. The use of NSI during ETS seems to arise from conventional knowledge such as experience and traditional practice, not scientific evidence or research. ${ }^{[2,3]}$

In South Africa (SA), the practice of NSI appears to continue even where protocols for ETS have been amended to recommend nonuse of NSI. NSI is implemented despite the associated risks and the fact that it is not recommended by current literature for safer ETS. ${ }^{[2,2,13]}$ Considering the lack of support for the benefits of NSI, recommendations and risks associated with NSI, and its routine implementation in many SA ICUs, this study was conducted to determine the effect of NSI on cardiorespiratory parameters in intubated cardiothoracic patients.

\section{Methods Design}

A prospective, randomised and comparative design was used to achieve the research objectives.

\section{Population, sampling and sample size}

The study was conducted in a surgical ICU of a private hospital in Gauteng that admits different surgical patients after coronary artery bypass graft (CABG), valve annuloplasty, repair or replacement, 
heart or lung transplant, thoracotomies and thoracic aneurysm repairs.

The target population included all patients in the ICU who had cardiac surgery, including patients who were on cardiopulmonary bypass during open heart surgery $(N=65)$. The following inclusion criteria applied: adults $>18$ years of age, intubation and mechanical ventilation, presence of an arterial line, continuous electrocardiograph and pulse oximetry $\left(\mathrm{SpO}_{2}\right)$ monitoring. Simple random sampling was used to assign patients to their respective groups: Group 1 comprised patients who were suctioned without NSI $(n=32)$; while group 2 comprised patients who were suctioned with NSI ( $n=33)$. Every second patient was assigned to group 1 after the first patient was assigned into group 2 . The operating theatre lists were used as the sampling frame.

Patients' demographic data, baseline vital data and data on treatments considered for their possible extraneous influence were collected according to standard procedure
Table 1. Demographic and baseline data of the sample $(N=65)$

\begin{tabular}{|c|c|c|c|c|c|c|}
\hline \multirow[b]{2}{*}{ Demographic data } & \multicolumn{2}{|c|}{ Total sample } & \multicolumn{2}{|c|}{$\begin{array}{l}\text { Group } 1 \\
\text { (non-NSI) }\end{array}$} & \multicolumn{2}{|c|}{$\begin{array}{l}\text { Group } 2 \\
\text { (NSI) }\end{array}$} \\
\hline & $n$ & $\%$ & $n$ & $\%$ & $n$ & $\%$ \\
\hline \multicolumn{7}{|l|}{ Age (years) } \\
\hline $20-39$ & 11 & 16.92 & 6 & 18.76 & 5 & 15.15 \\
\hline $40-59$ & 24 & 36.92 & 11 & 34.38 & 13 & 39.39 \\
\hline $60-79$ & 26 & 40.00 & 13 & 40.63 & 13 & 39.39 \\
\hline$>80$ & 4 & 6.15 & 2 & 6.25 & 2 & 6.06 \\
\hline \multicolumn{7}{|l|}{ Gender } \\
\hline Male & 41 & 63.08 & 19 & 59.39 & 22 & 66.67 \\
\hline Female & 24 & 36.92 & 13 & 40.63 & 11 & 33.33 \\
\hline \multicolumn{7}{|l|}{ Smoking history } \\
\hline No & 52 & 80.00 & 28 & 87.50 & 24 & 72.73 \\
\hline Yes & 13 & 20.00 & 4 & 12.50 & 9 & 27.27 \\
\hline \multicolumn{7}{|l|}{ Surgery } \\
\hline Aortic valve replacement & 10 & 15.38 & 6 & 18.75 & 4 & 12.12 \\
\hline Coronary artery bypass graft & 36 & 55.39 & 18 & 56.25 & 18 & 54.55 \\
\hline Double valve replacement & 4 & 6.15 & 1 & 3.13 & 3 & 9.09 \\
\hline Mitral valve repair & 13 & 20.00 & 7 & 21.88 & 6 & 18.18 \\
\hline Pericardectomy & 1 & 1.54 & - & - & 1 & 3.03 \\
\hline Repair ventricular septal defect & 1 & 1.54 & - & - & 1 & 3.03 \\
\hline \multicolumn{7}{|l|}{$\mathrm{FiO}_{2}$} \\
\hline 0.4 & 39 & 60.00 & 22 & 68.75 & 17 & 51.52 \\
\hline 0.5 & 12 & 18.46 & 5 & 15.63 & 7 & 21.21 \\
\hline 0.6 & 14 & 21.54 & 5 & 15.63 & 9 & 27.27 \\
\hline \multicolumn{7}{|l|}{ RR (breaths/minute) } \\
\hline 10 & 6 & 9.23 & 4 & 12.50 & 2 & 6.06 \\
\hline 12 & 33 & 50.77 & 13 & 40.63 & 20 & 60.61 \\
\hline 14 & 17 & 26.15 & 9 & 28.13 & 8 & 24.24 \\
\hline 16 & 9 & 13.85 & 6 & 18.75 & 3 & 9.09 \\
\hline \multicolumn{7}{|l|}{ Inotropes } \\
\hline Yes & 12 & 18.46 & 6 & 18.75 & 6 & 18.18 \\
\hline No & 53 & 81.54 & 26 & 81.25 & 27 & 81.25 \\
\hline \multicolumn{7}{|l|}{ Sedated/paralysed } \\
\hline No & 28 & 43.08 & 13 & 40.63 & 15 & 45.45 \\
\hline Yes & 37 & 56.08 & 19 & 59.38 & 18 & 54.55 \\
\hline
\end{tabular}

$\mathrm{NSI}=$ normal saline instillation; $\mathrm{FiO}_{2}=$ fraction of inspired oxygen; $\mathrm{RR}=$ respiratory rate from patients' ICU charts and admission records. Measurements of primary variables - peripheral oxygen saturation $\left(\mathrm{SpO}_{2}\right)$, arterial blood gas, HR and BP - were obtained 5 minutes pre suctioning and again at 1-, 5-, 10-, 30- and 60-minute intervals, post suctioning. Arterial blood gases were measured 5 minutes pre suctioning and 60 minutes post suctioning only. Patients were suctioned with or without NSI 6 hours post surgery and then $4-6$ hours thereafter as per unit protocol. Patients were enrolled in the study for their first suctioning event, i.e. 6 hours post surgery.

\section{Group 1: without NSI}

Suctioning was performed according to standard principles. The patient was preoxygenated for a period of 1 minute before the procedure. Then, using a $14 \mathrm{Fr}$ catheter, the patient was suctioned for 8 seconds. Only three suctioning passes were made using an open suctioning technique in a single suctioning episode. After the suctioning procedure, the patient was again pre-oxygenated for a period of 1 minute. Then the oxygen setting was turned down on the ventilator before the $\mathrm{SpO}_{2}$ measurement was recorded.

\section{Group 2: with NSI}

The patient was pre-oxygenated for a period of 1 minute before the procedure. Then $5 \mathrm{~mL}$ of normal saline was instilled into the ET and the patient was reconnected to the ventilator and given a few breaths. A $14 \mathrm{Fr}$ catheter was used to perform the suctioning procedure for 8 seconds. The same procedures were carried out after suctioning, as was done with patients who did not receive NSI.

\section{Instrument}

Data were captured onto a researcherdeveloped tool based on a recent literature review. The data-capturing tool comprised four sections: (i) patients' demographic data: age, gender, smoking history and surgical procedure; (ii) baseline vital data: patients' fraction of inspired oxygen concentration $\left(\mathrm{FiO}_{2}\right)$ and mandatory respiratory rate (RR); (iii) treatment: presence/absence of use of inotropes and sedatives/paralytic agents; (iv) study variables: $\mathrm{SpO}_{2}, \mathrm{HR}, \mathrm{pH}$, partial pressure of oxygen $\left(\mathrm{PaO}_{2}\right)$, serum bicarbonate level $\left(\mathrm{HCO}_{3}^{-}\right)$, arterial oxygen saturation as measured by blood gas analyser $\left(\mathrm{SaO}_{2}\right)$, systolic BP (SBP), diastolic BP (DBP) and mean arterial pressure (MAP). Codes were 
used instead of participants' names to ensure anonymity and confidentiality.

\section{Data analysis}

Descriptive statistics were used to present and analyse the data; where applicable percentages, means and standard deviations (SDs) were used to summarise results. Fisher's Exact and $\chi^{2}$ tests were applied for all categorical variables and the two-sample $t$-test was used to test for the difference between the means of the two study groups. Testing was done at $p<0.05$.

\section{Ethical considerations}

The study was approved by the University of the Witwatersrand Human Research Ethics Committee (Medical) (reference M030976). In the hospital, permission to conduct the study was given by the Provincial Health Directorate, the hospital chief executive and the ICU managers. Participation was voluntary and patient participants signed a written informed consent preoperatively before inclusion in the study.

\section{Results}

Of the total sample, $41(63 \%)$ were males and $24(37 \%)$ were females in the age range 20 - 80 years. The mean (SD) age for group 1 was 55 (14) and 56 (14) years for group 2. Non-smokers dominated the sample $(n=52$; $80 \%$ ) and were evenly distributed between the groups. The majority of the patients underwent coronary artery bypass graft (CABG) (55.39\%), 13 patients (20.00\%) underwent mitral valve repair, and 10 patients $(15.38 \%)$ underwent aortic valve replacement.

Baseline data of the sample are summarised in Table 1. At the time of observation (5 minutes pre suctioning) 39 patients (60\%) were on $0.4 \mathrm{FiO}_{2}, 12$ (18\%) patients on $0.5 \mathrm{FiO}_{2}$ and 14 patients (22\%) on $0.6 \mathrm{FiO}_{2}$. The $\mathrm{FiO}_{2}$ was left constant throughout the period of observation. A larger percentage of patients being on $0.4 \mathrm{FiO}_{2}$ may indicate that the majority of patients did not have any problems with oxygenation. More than half $(n=33 ; 51 \%)$ of the patients were ventilated on a rate of 12 breaths/ minute $(b / \mathrm{min}), 26 \%(n=17)$ were on a rate of $14 \mathrm{~b} / \mathrm{min}, 14 \%(n=9)$ on a rate of $16 \mathrm{~b} / \mathrm{min}$ and $9 \%(n=6)$ on a rate of $10 \mathrm{~b} /$ min. Mean ventilation rate for group 1 was $13(2.4) \mathrm{b} / \mathrm{min}$, and for group 2 was $13(1.5) \mathrm{b} / \mathrm{min}$. The majority of patients $(n=53 ; 82 \%)$ were on inotropes: more than half $(n=37 ; 57 \%)$ received some form of sedation; 59\% $(n=19)$ and $55 \%(n=18)$ were in groups 1 and 2, respectively. There was no difference in the baseline variables, indicating that the two groups were comparable.

\section{Peripheral oxygen saturation $\left(\mathrm{SpO}_{2}\right)$}

$\mathrm{SpO}_{2}$ was observed at 5 minutes presuctioning and at 1, 5, 10, 30 and 60 minutes post suctioning for groups 1 and 2 (Fig. 1).

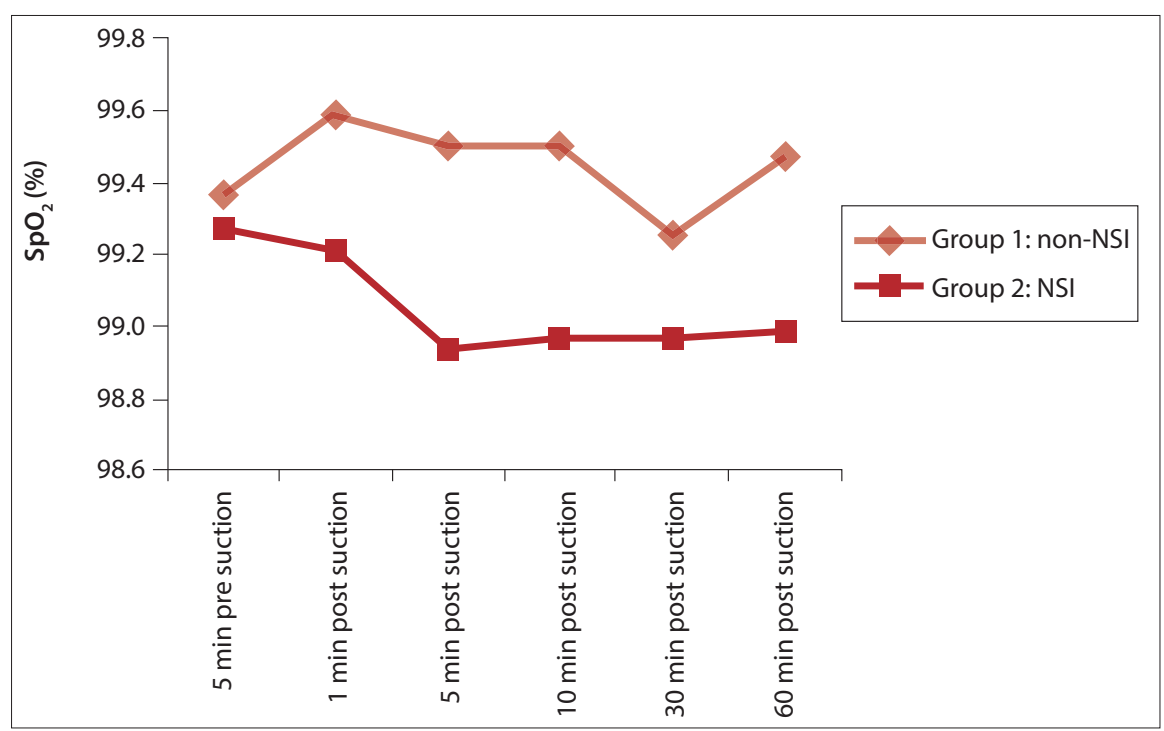

Fig. 1. Comparison of $\mathrm{SpO}_{2}$ with non-use (non-NSI) and use (NSI) of normal saline. $\left(\mathrm{SpO}_{2}=\right.$ arterial oxygen saturation as measured by pulse oximetry; NSI = normal saline instillation.)

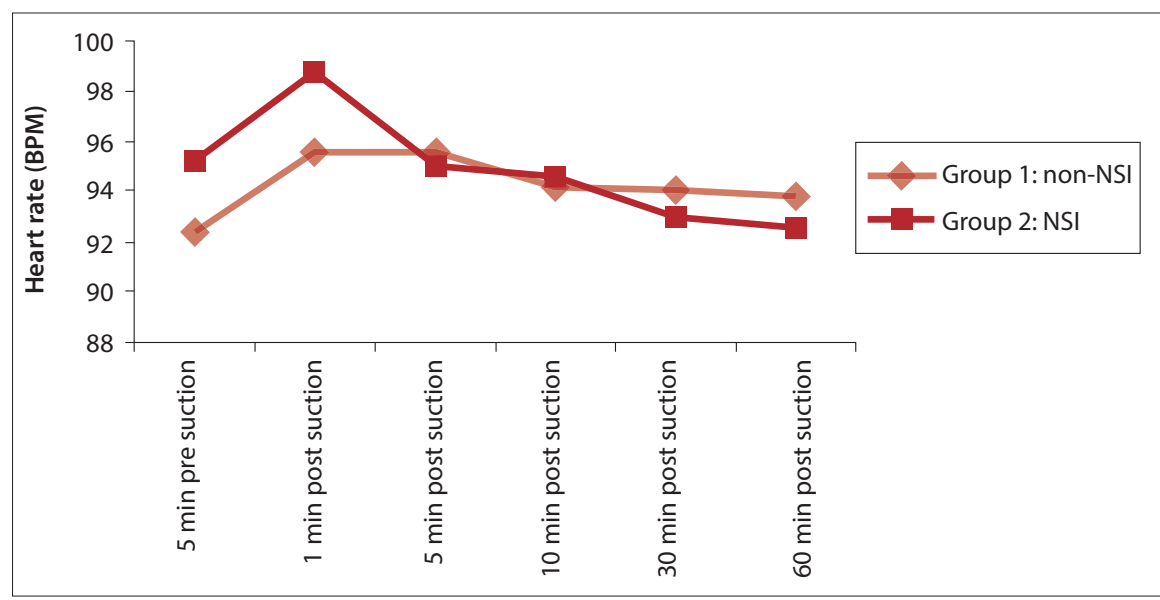

Fig. 2. The effect of heart rate with non-use (non-NSI) and use (NSI) of normal saline. (BPM = beats per minute; NSI = normal saline instillation.)

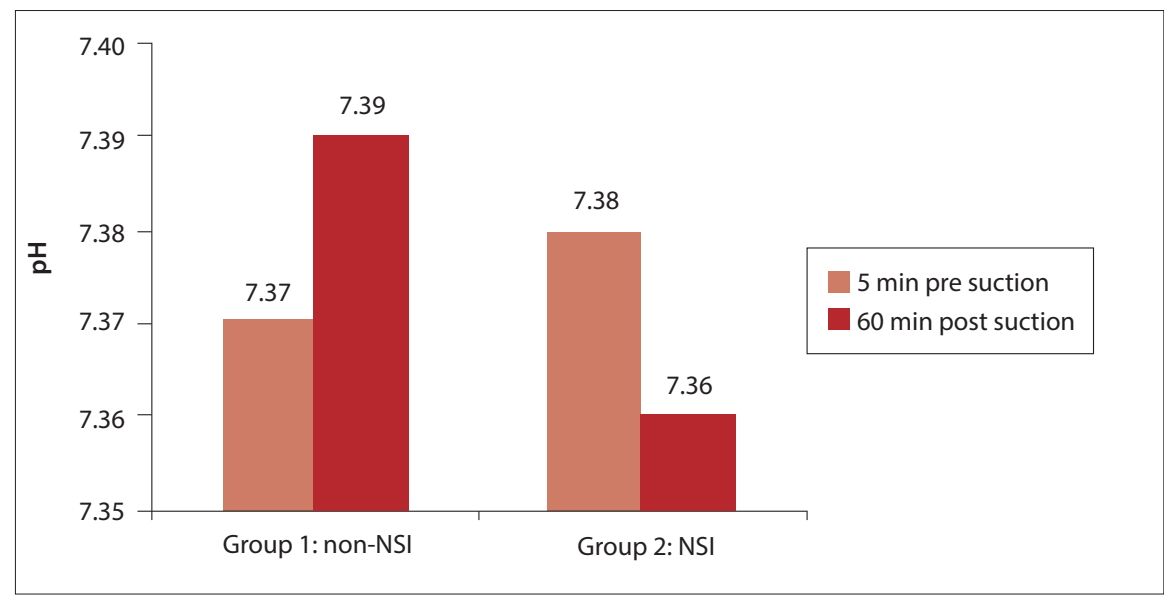

Fig. 3. Comparison of pH values with non-use (non-NSI) and use (NSI) of normal saline. (NSI = normal saline instillation.) 
A positive difference between $\mathrm{SpO}_{2}$ at different observation times post suctioning indicates an increase in $\mathrm{SpO}_{2}$ while a negative difference indicates a decrease in $\mathrm{SpO}_{2}$. These results suggest that there was an increase in $\mathrm{SpO}_{2}$ at all observation times except at 30 minutes post suctioning in group 1 and a decrease in $\mathrm{SpO}_{2}$ at all observation times in group 2. Although these differences existed, they were not statistically significant as evidenced by the $p$-values.

\section{Heart rate (HR)}

HR was monitored at 5 minutes pre suctioning, and at 1, 5, 10, 30 and 60 minutes post suctioning for both groups 1 and 2 . There was an overall increase in $\mathrm{HR}$ at all observation times in group 1 and an overall decrease in group 2 except at 1 minute. Again these changes were not statistically significant (Fig. 2).

\section{Acid base status $(\mathrm{pH})$}

The $\mathrm{pH}$ was examined at 5 minutes pre suctioning and 60 minutes post suctioning for both groups. The $\mathrm{pH}$ mean at 5 minutes was $7.37(0.06)$ for group 1 and 7.38 (0.07) for group 2, while at 60 minutes the $\mathrm{pH}$ was 7.39 (0.51) for group 1 and 7.36 (0.05) for group 2 (Fig. 3). This increase in $\mathrm{pH}$ (by 0.01 ) in group 1 and decrease in $\mathrm{pH}$ (by 0.03 ) in group 2 was statistically significant at $p=0.1$ (within group 1), $p=0.02$ (within group 2) and $p=0.04$ (difference between groups) post suctioning. Similarly, the difference between pre- and postsuction $\mathrm{pH}$ levels for group 2 was significant $(p>0.05)$, suggesting that the drop in $\mathrm{pH}$ in group 2 was statistically significant compared with the non-significant increase in $\mathrm{pH}$ in group 1 .

\section{Partial pressure of oxygen $\left(\mathrm{PaO}_{2}\right)$}

The data in Fig. 4 show that there was a clear decrease in $\mathrm{PaO}_{2}$ after the procedures. A $t$-test was used to compare $\mathrm{PaO}_{2}$ means within groups and between groups. In both groups, there was a decrease in $\mathrm{PaO}_{2}$ post suctioning (-4.1 mmHg for group $1 \mathrm{v} .-13.73$ $\mathrm{mmHg}$ for group 2). These differences were not statistically significant. It can be noted that the decrease in $\mathrm{PaO}_{2}$ was greater in group $2(11 \%)$ than in group 1 (3\%).

\section{Standard bicarbonate $\left(\mathrm{HCO}_{3}^{-}\right)$}

When the $\mathrm{HCO}_{3}^{-}$levels with use of normal saline and non-use of normal saline were examined, there was a slight increase in

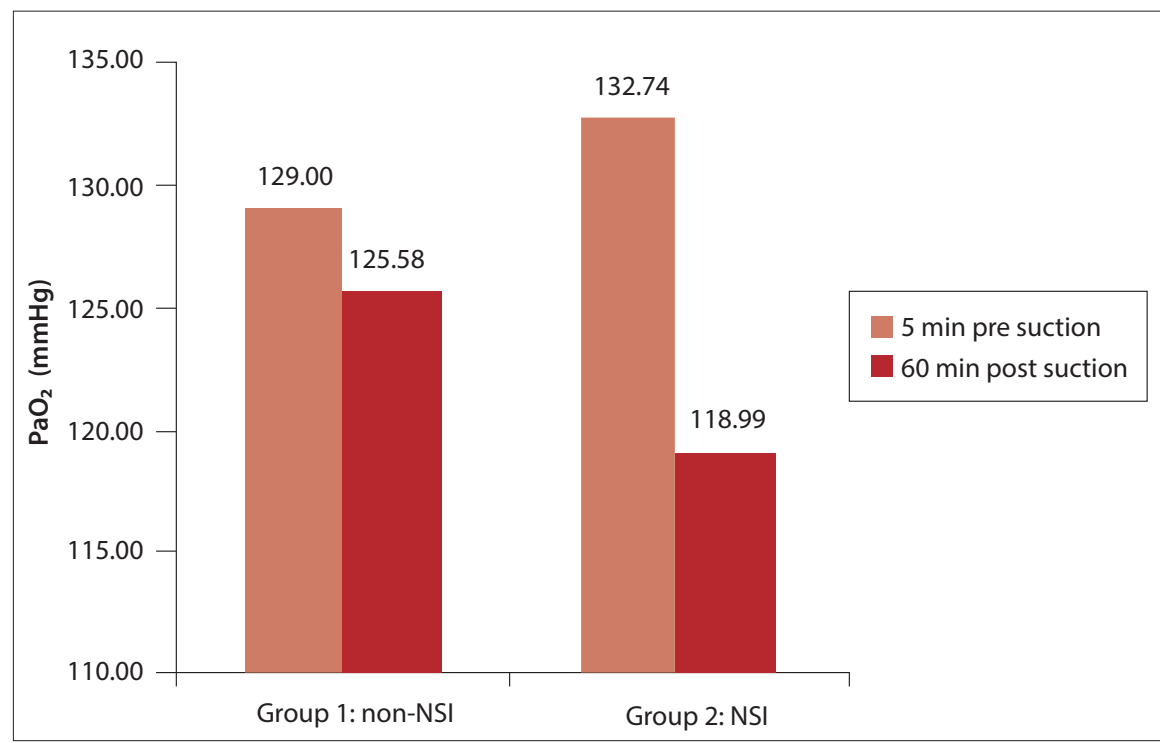

Fig. 4. Comparison of $\mathrm{PaO}_{2}$ with non-use (non-NSI) and use (NSI) of normal saline. $\left(\mathrm{PaO}_{2}=\right.$ arterial partial pressure of oxygen; NSI = normal saline instillation.)

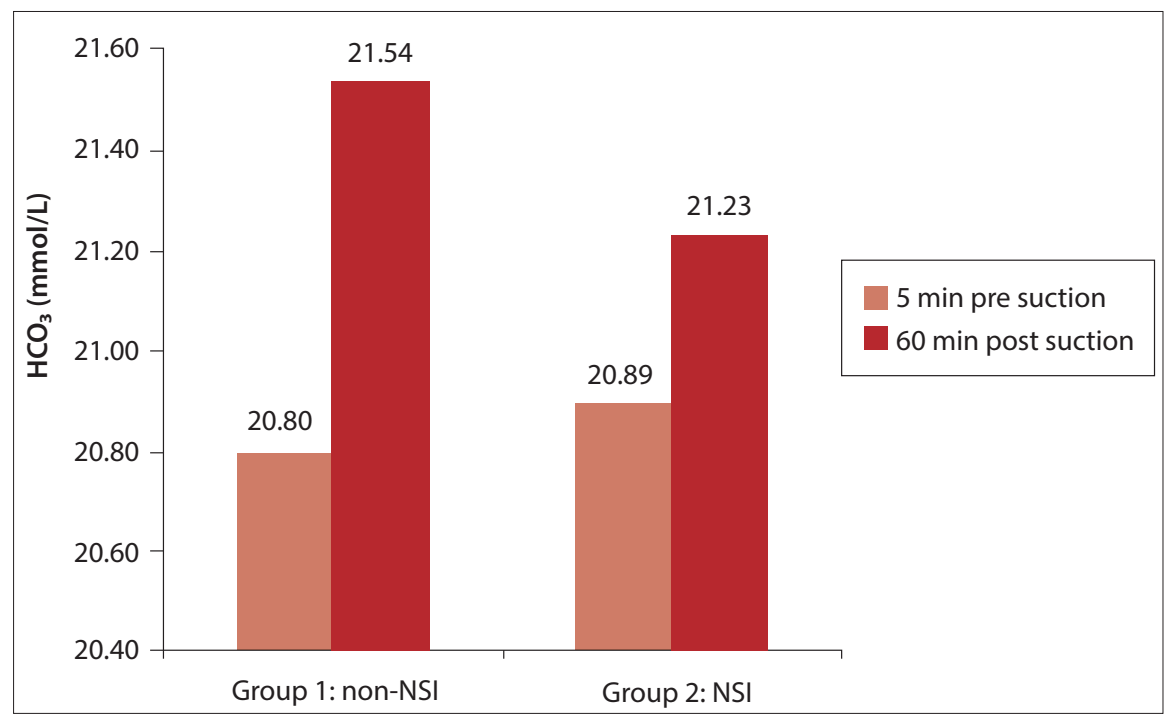

Fig. 5. Comparison of $\mathrm{HCO}_{3}$ levels with non-use (non-NSI) and use (NSI) of normal saline. (NSI = normal saline instillation.)

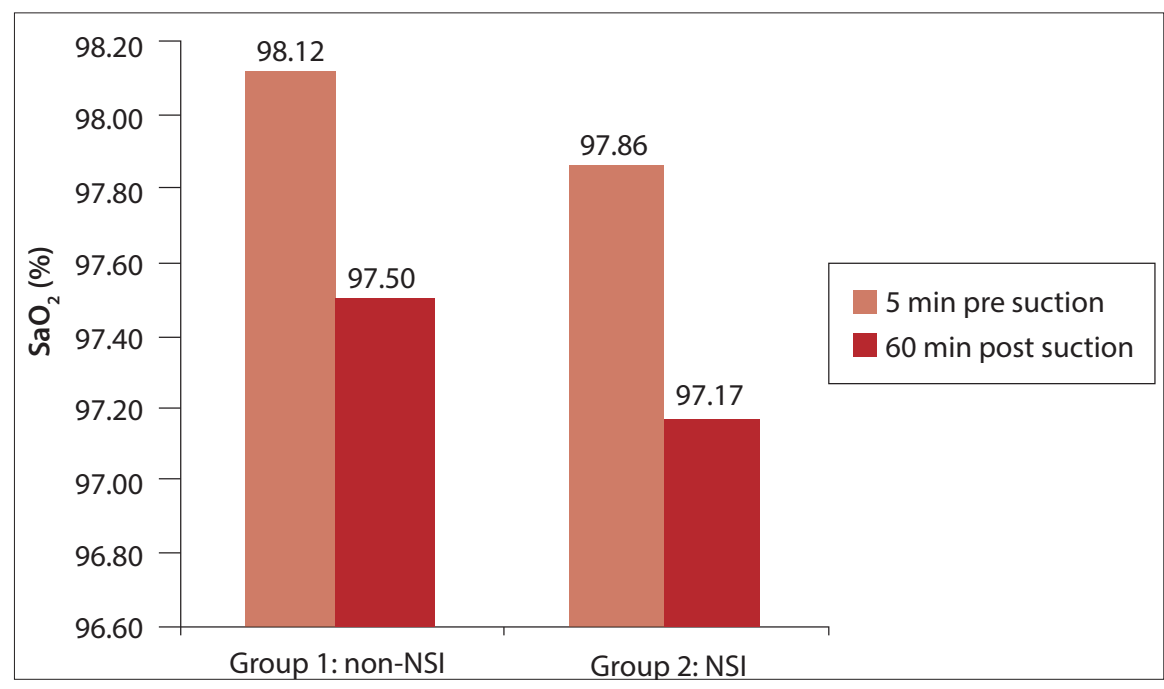

Fig. 6. Comparison of $\mathrm{SaO}_{2}$ values with non-use (non-NSI) and use (NSI) of normal saline. $\left(\mathrm{SaO}_{2}=\right.$ arterial oxygen saturation as measured by blood gas analyser; NSI = normal saline instillation.) 


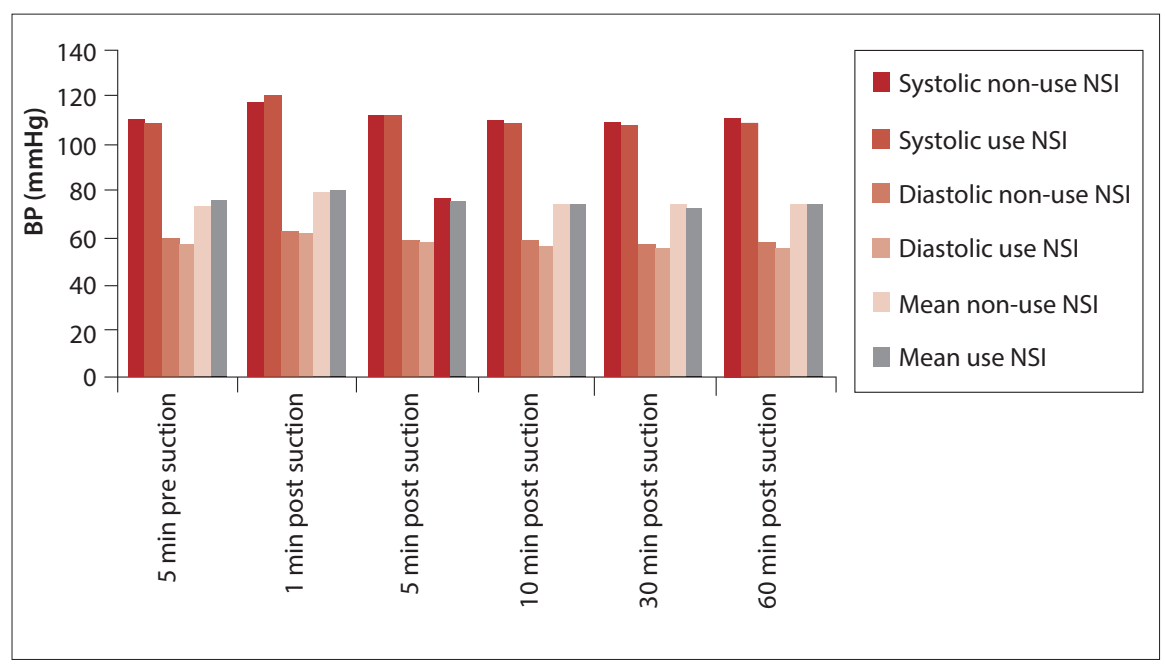

Fig. 7. The effect of BP (systolic, diastolic and mean) with non-use and use of normal saline. $(B P=$ blood pressure; NSI = normal saline instillation. $)$

$\mathrm{HCO}_{3}^{-}$in both groups, i.e. $0.7 \mathrm{mmol} / \mathrm{L}$ in group 1 v. $0.3 \mathrm{mmol} / \mathrm{L}$ in group 2 (Fig. 5). These changes and differences were not statistically significant.

\section{Arterial oxygen saturation $\left(\mathrm{SaO}_{2}\right)$}

ETS resulted in a decrease in $\mathrm{SaO}_{2}$ levels. $\mathrm{SaO}_{2}$ was monitored at 5 minutes and 60 minutes pre suctioning for both groups. A decrease in $\mathrm{SaO}_{2}$ occurred in both groups $(-0.6 \%$ in group 1 and $-1.7 \%$ in group 2) (Fig. 6). The changes and differences detected were not statistically significant.

BP (SBP, DBP and MAP) increased immediately (1 minute post suctioning) and over time returned to the baseline readings for both groups (Fig. 7). All $p$-values were $>0.05$, suggesting that these differences were not statistically significant.

\section{Discussion}

This study investigated the effects of NSI on cardiorespiratory parameters in intubated cardiothoracic patients. Patients were similar with respect to demographic and baseline data (mode of ventilation and RR) and treatment (presence/absence of use of inotropes and sedatives/paralytic agents). No significant differences were found when groups were compared in respect of these characteristics. These early postelective surgery patients were unlikely to have excessive or infected secretions.

NSI was accompanied by a slight decrease $(-0.1-0.3 \%)$ in $\mathrm{SpO}_{2}$ for up to 60 minutes post suctioning. When compared with baseline, this finding is supported in the study of Akgül et al. ${ }^{[10]} \mathrm{A}$ secondary analysis was done to compare groups in respect of the return rate of $\mathrm{SpO}_{2}$ to baseline level at 30 minutes post suctioning. These differences were statistically significant, suggesting that the use of NSI during ETS negatively influences oxygenation, which supports the growing literature against the use of NSI. The literature has suggested that NSI interferes with alveolar capillary oxygen exhange, resulting in decreased oxygen saturation. ${ }^{[14]}$ This study did not observe whether the patients who failed to return to baseline $\mathrm{SpO}_{2}$ after 30 minutes post suctioning ever returned to baseline at all or if they did return, how long it took.

When HR was observed in this study, it was found that HR means were slightly increased at 1 minute post suctioning with and without NSI, unlike the Akgül et $a{ }^{\left[{ }^{10]}\right.}$ study where HR only increased at 4 - 5 minutes in the postsuctioning NSI group. In this study, HR mean pressures were increased until 60 minutes post decreased from 5 minutes to 60 minutes post suctioning in the NSI group. This difference was statistically significant in the Akgül et al. study, ${ }^{[10]}$ while in this study no statistically significant differences were found.

All three aspects of BP (SBP, DBP and MBP) were addressed in this study. It was found that there was an increase in BP immediately post suctioning in both groups, and over time the BP stabilised towards baseline value. The increase in BP immediately post suctioning may be attributed to stress and anxiety caused by the ETS procedure itself rather than the effects of NSI. Thus a factor other than NSI such as inadequate patient preparation may have led to $\mathrm{BP}$ increase. No suctioning in the non-NSI group, while they previously published studies seem to have addressed the effects of ETS with or without NSI on BP.

Together with $\mathrm{SpO}_{2}, \mathrm{PaO}_{2}$ and $\mathrm{SaO}_{2}$ are direct measures of oxygenation. No statistically significant differences were found when groups were compared in respect of $\mathrm{PaO}_{2}$ and $\mathrm{SaO}_{2}$. It was noted that the decrease in $\mathrm{PaO}_{2}$ was greater in the NSI group (11\%) than the non-NSI group (3\%). These results correlate to the findings by Bostick and Wendelgass. ${ }^{[14]}$ In their study, the differences in $\mathrm{PaO}_{2}$ before and after suctioning were $4 \mathrm{mmHg}$ in the non-NSI group, $11.7 \mathrm{mmHg}$ in the $5 \mathrm{~mL} \mathrm{NSI} \mathrm{group}$ and $13.5 \mathrm{mmHg}$ in the $10 \mathrm{~mL}$ NSI group. They attributed this decrease to the idea that NSI impedes alveolar capillary gas exchange. However they recommended replication of the study with refinement of methodology. Corresponding results were found despite variation in methodology between this study and that of Bostick and Wendelgass, ${ }^{[14]}$ i.e. both results suggest NSI may have a negative effect on oxygenation. A small variation in $\mathrm{SaO}_{2}$ was found pre and post suctioning with or without NSI. In both cases, there was a slight decrease in the $\mathrm{SaO}_{2}$ means after suctioning $(-0.6 \%$ decrease in the non-NSI group and $0.7 \%$ decrease in the NSI group). These differences in $\mathrm{SaO}_{2}$ were not statistically significant.

As part of the arterial blood gases, $\mathrm{pH}$ and $\mathrm{HCO}_{3}^{-}$were assessed in this study. There was a decrease in $\mathrm{pH}$ when normal saline was used compared with an increase in $\mathrm{pH}$ when not used; the decrease in $\mathrm{pH}$ in the NSI group was found to be statistically significant. Statistically significant results were also found when $\mathrm{pH}$ values were compared between groups. In the study by the Akgül et al., ${ }^{[10]}$ the rise in $\mathrm{pH}$ when NSI was used was statistically significant; however, the differences in $\mathrm{pH}$ were not statistically significant between groups. The results do not correspond to the findings of this study.

The change in $\mathrm{pH}$ could have been due to interruption of ventilation during suctioning or other factors not related to suctioning. This study did not address the effects of ETS on $\mathrm{CO}_{2}$ with or without NSI. Akgül et al. found no significance difference in $\mathrm{CO}_{2}$ when NSI was used or not used during suctioning. In this study, there were small increases in $\mathrm{HCO}_{3}^{-}$levels before and after suctioning with use of normal saline $(0.7 \mathrm{mmol} / \mathrm{L}$ in group 1 and $0.3 \mathrm{mmol} / \mathrm{L}$ in group 2). Presumably this could also be related to the decrease in $\mathrm{pH}$. 
These differences were not statistically significant in this study and correspond to the findings of Akgül et al. ${ }^{[10]}$

\section{Study limitations}

The findings of this study are limited to a homogenous population of adults who underwent open heart surgery and were ventilated for short-term purposes. The sample size was reduced from 74 to $65 ; 74$ was initially the appropriate sample for this study according to statistical determination. Due to lack of subjects volunteering, only 65 patients were included in the study after 8 months of data collection. We acknowledge that the small sample size may have influenced the ability of this study to generate sufficient statistical significance. In hindsight we see another limitation in the fact that our data collection tool was developed from the literature and as such the effect of $\mathrm{PaCO}_{2}$ level was not captured in this study. Furthermore, this study did not observe the return rate of the $\mathrm{SaO}_{2}$ to baseline after ETS. Based on our findings, we can hypothesise that $\mathrm{SaO}_{2}$ may be slow to recover in the NSI group. We recommend that this be followed up and included in future studies.

\section{Conclusions}

It can be concluded that NSI has no effect on cardiorespiratory parameters in intubated cardiothoracic patients. Yet the literature suggests that NSI has adverse effects on physiological parameters. ${ }^{[4,5]}$ The only extrapolation that can be made from this study is that further studies need to be done. We can, however, speculate that myocardial ischaemia in CABG patients is unlikely to be precipitated by suctioning or instilling normal saline. Our findings could have been affected by beta blockade in the CABG patients.
References

1. Hyunsoo $\mathrm{O}$, Whasook $\mathrm{S}$. A meta-analysis of the effects of various interventions in preventing endotracheal suction-induced hypoxemia. J Clin Nurs 2003;12(6):912-924

2. Day T, Farnell S, Haynes S, Wainwright S, Wilson-Barnett J. Tracheal suctioning: An exploration of nurses' knowledge and competence in acute and high dependency ward areas. J Adv Nurs 2002;39(1):35-45.

3. Pedersen C, Rosendahl-Nielsen M, Hjermind J, Egerod I. Endotracheal suctioning of the adult intubated patient - What is the evidence? Intensive Crit Care Nurs 2009;25(1):21-30. [http:// dx.doi.org/10.1016/j.iccn.2008.05.004]

4. Kelleher $\mathrm{S}$, Andrews T. An observational study on the open-system endotracheal suctioning practices in critical care nurses. J Clin Nurs 2008;17(3):360-369. [http://dx.doi.org/10.1111/ J.1365-2702.2007.01990.x

5. Celik S, Kanan N. A current conflict: Use of isotonic sodium chloride on endotracheal suctioning in critically ill patients. Dimens Crit Care Nurs 2006;25(1):11-14.

6. Caruso $P$, Denari $S$, Ruiz $S$, Demarzo $S$, Deheinzelin $D$. Saline instillation before trachea suctioning decreases the incidence of ventilator-associated pneumonia. Crit Care Med 2009;37(1):32-38. [http://dx.doi.org/10.1097/CCM.0b013e3181930026]

7. Topeli A, Harmanci A, Cetinkaya Y, Akdeniz S, Unai S. Comparison of the effect of closed endotracheal suction systems on the development of ventilator-associated pneumonia. endotracheal suction systems on the development of ventilator-associated

8. Riddling D, Martin L, Bratton S. Endotracheal suctioning with or without instillation of isotonic sodium chloride solution in critically ill children. Am J Crit Care 2003;12(3):212-219.

9. O'Neal P, Grap J, Thompson C, Dudley N. Level of dyspnoea experienced in mechanically ventilated adults with and without normal saline instillation prior to endotracheal suctioning. Intensive Crit Care Nurs 2001:17:356-363.

10. Akgül S, Akyolcu N. Effects of normal saline on endotracheal suctioning. J Clin Nurs 2002;11(6):826-830. [http://dx.doi.org/10.1046/j.1365-2702.2002.00655.x]

11. Seckel MA. Is this research ready to be translated into practice? AACN Adv Crit Care 2009;20(4):322-324. [http://dx.doi.org/10.1097/NCl.0b013e3181ae1049]

12. American Association of Respiratory Care (AARC). AARC Clinical Practice: Endotracheal suctioning of mechanically ventilated adults and children with artificial airways. Respir Care 1993;38(4):5000-5004.

13. Lynn-Mchlane D, Carlson K. AACN Procedure Manual for Critical Care. 5th edition. St Louis: Saunders Elsevier, 2005.

14. Bostick J, Wendelgass S. Normal saline instillation as part of the suctioning procedure. Effects on PaO2 and amount of secretions. Heart Lung 1987;16(5):532-537.

15. Ackerman M, Mick D. Installation of normal saline before suctioning in patients with normal pulmonary infections: A prospective randomised controlled trial. Am J Crit Care 1998;7(4):261-266.

16. Shorten $D$, Bryne $P$, Jones R. Infant responses to normal saline institutions and endotracheal suctioning. J Obstet Gynecol and Neonatal Nurs 1991;20(6):464-469.

17. EndacottR, Botti M. Clinical research 3: sample selection. Intensive Crit Care Nurs 2005;21(1):51 55. [http://dx.doi.org/10.1016/j.iccn.2004.11.001]

18. Blackwood B. Normal saline instillation with endotracheal suctioning: Primum non nocere (first do no harm). J Adv Nurs 1999:29(4):928-934. 